\title{
Tecnologias de Informação e Comunicação e Ensino Semipresencial na Educação Médica
}

\section{Information and Communication Technology and Blended Learning in Medical Education}

Ekaterini Simões Goudouris ${ }^{I}$ Taís Rabetti Giannella ${ }^{I}$ Miriam Struchiner

\section{KEYWORDS:}

- Educational Technology;

- Internet;

- Medical Education.
Recebido em: 15/06/2012

Reencaminhado em: 08/03/2013

Aprovado em: 20/08/2013

REVISTA BRASILEIRA DE EDUCAÇÃO MÉDICA

\section{RESUMO}

O ensino médico vem enfrentando desafios para modificar suas práticas pedagógicas e estrutura curricular. As tecnologias de informação e comunicação (TICS) podem auxiliar no enfrentamento de muitos desses desafios. Tendo em vista esse cenário, este estudo tem como objetivo caracterizar as formas de apropriação de uma Ferramenta de Autoria de cursos na Internet em processos formativos semipresenciais de graduação, pós-graduação, educação continuada e extensão na área médica, no contexto da UFRJ. Com base nas análises, foi possível identificar o perfil de utilização das TICs e discutir os resultados, a partir dos ambientes virtuais de aprendizagem construídos pelos professores, de acordo com as seguintes categorias: unidades acadêmicas de origem; disciplinas e níveis de ensino envolvidos; configuração de áreas de conhecimento dos cursos semipresenciais; conformação das equipes docentes dos cursos; recursos de conteúdo e de comunicação utilizados; e atividades pedagógicas adotadas.

Medical education is facing challenges to modify teaching practices and curriculum structure. Information and Communication Technology (ICT) can facilitate tackling several of these challenges. In this scenario, this study aimed at identifying forms of adopting an Internet based course authoring tool in blended learning formative processes in undergraduate, graduate, and continuing medical education at UFRJ. Based on the analyses it was possible to identify a profile of ICT usage and to discuss the results according to the following categories derived from the Virtual Learning Environments developed by professors: academic unit affiliation, disciplines and educational levels involved, field of knowledge of the blended learning courses, formation of the teaching staff team for the courses, content and communication resources used and educational activities adopted. 


\section{INTRODUÇÃO}

Muitos são os desafios enfrentados pela educação médica, dentre eles destaca-se sua conformação disciplinar. A prática médica requer conhecimentos de diferentes áreas, desde as biociências e ciências clínicas até as humanas e sociais, que vêm assumindo cada vez mais um papel fundamental na compreensão sobre os processos de saúde-doença, conferindo à medicina conhecimentos de natureza interdisciplinar. Desse modo, o ensino médico vem sendo desafiado a dar conta não apenas de uma sólida formação técnico-científica, mas de incorporar, nessa formação, uma abordagem humanizada e contextualizada, em uma perspectiva de integralidade e de universalidade da atenção à saúde da população ${ }^{1,2,3}$. A discussão acerca da educação médica não se limita à formação na graduação, mas também na pós-graduação lato sensu (especialização e residência médica) e stricto sensu (mestrado e doutorado), a educação continuada e permanente ${ }^{4}$.

A partir da publicação do relatório Flexner, em 1910, amplas reformas foram implementadas no ensino médico. Contudo, segundo avaliação do próprio autor realizada 15 anos depois da publicação do documento, as mudanças produziram maior valorização dos aspectos científicos da medicina, em detrimento de seus aspectos humanísticos e sociais ${ }^{5,6}$. Portanto, embora a educação médica já estivesse passando por modificações, houve a consolidação do modelo de ensino baseado no pensamento científico hegemônico ${ }^{7}$, como uma formação biologicista, fragmentada em disciplinas, focada em superrespecialização precoce e alicerçada em processos de avaliação cognitiva com base no acúmulo de conteúdos técnico-científicos ${ }^{8}$.

Apesar desse modelo ainda ser o hegemônico, nas últimas décadas o ensino médico vem sendo questionado quanto às suas práticas pedagógicas e estrutura curricular devido, por um lado, às profundas mudanças na sociedade ${ }^{4}$ e nos paradigmas da educação e da saúde, que buscam uma educação emancipatória, crítica, centrada no aluno por meio de metodologias ativas 9,10,11 com o construtivismo e o construtivismo sociocultural como marcos pedagógicos ${ }^{12}$; por outro, à definição de saúde da Organização Mundial da Saúde (OMS) como bem-estar físico, mental e social e o movimento preventivista ${ }^{13}$, que redefinem a abordagem para a formação em saúde.

As instituições de ensino superior vêm, portanto, sendo pressionadas a rever não apenas seus processos de formação, como também seu relacionamento com a sociedade ${ }^{14}$. Sociedade, esta em que as tecnologias de informação e comunicação (TICs) estão em crescente difusão, impactando os ambientes de ensino-aprendizagem, particularmente as formas de comunicação entre seus principais atores: professores e alunos ${ }^{15}$.
As TICs oferecem ferramentas que podem trazer contribuições à implementação de novos modelos pedagógicos, a partir do acesso facilitado a conteúdos de ensino em formatos variados (textos, apresentações, vídeos, animações) ${ }^{16}$, do favorecimento da autonomia dos alunos ${ }^{17}$, bem como da possibilidade de criação de novos canais de comunicação entre alunos e professores, e entre os alunos entre si e com a comunidade $^{18}$. O que se observa é que as TICs têm produzido transformações nas formas de praticar e de ensinar Medicina, para dar conta de necessidades específicas da formação profissional na graduação, na pós-graduação e na educação permanente $^{19,20}$.

No Brasil, é crescente o número de programas voltados para a adoção de TICs na formação em saúde, especialmente nas estratégias de educação permanente ${ }^{21}$. Dentre eles, pode-se citar o Programa Telessaúde Brasil e a Rede de Telemedicina Universitária ${ }^{22}$. A consolidação da EaD como modalidade educacional no âmbito da saúde é reforçada com a criação da Universidade Aberta do Sistema Único de Saúde (UNA-SUS), voltada a atender as necessidades de formação e educação permanente dos trabalhadores do Sistema Único de Saúde (SUS) ${ }^{23}$

Entretanto, o uso da tecnologia em si não é garantia de sucesso nem de qualidade no processo de ensino-aprendizagem $^{24}$. Apesar das potencialidades anunciadas, é preciso considerar os problemas existentes na relação entre as TICs e a educação médica, tais como: inadequação dos modelos pedagógicos adotados; dificuldades de integração curricular; falta de familiaridade do docente com as TICs no ensino; e ausência de processos de avaliação adequados ${ }^{15}$.

No sentido de facilitar a disseminação do uso de TICs nas práticas pedagógicas em saúde, uma ferramenta de autoria de cursos na Internet, Constructore, foi desenvolvida na UFRJ, a partir de um processo de pesquisa marcado pela colaboração entre profissionais de diversas áreas (tecnologia educacional, informática, web design, professores-pesquisadores das ciências e saúde) e pela contínua construção de conhecimento e de formação contextualizada dos professores envolvidos, que se disponibilizaram a integrá-la em suas práticas educativas ${ }^{25}$.

É nesse cenário que este estudo se insere e tem como objetivo caracterizar as formas de apropriação da ferramenta Constructore $^{26}$ em processos formativos semipresenciais na área médica, no contexto da UFRJ. Pretende-se, assim, identificar o perfil de utilização das TICs nos seguintes aspectos: unidades acadêmicas; disciplinas e níveis de ensino envolvidos; configuração de áreas de conhecimento dos cursos; conformação das equipes docentes; e recursos e atividades pedagógicas adotados. 


\section{REVISÃO DA LITERATURA}

Na literatura sobre TICs no ensino superior, os termos AVA (Ambiente Virtual de Aprendizagem ou LVE - Learning Virtual Environments), CMS (Course Management Systems) e LMS (Learning Management Systems) são utilizados para se referir ao que se denomina ferramentas de autoria ${ }^{27}$. Estes sistemas têm por objetivo oferecer ferramentas para que gestores e professores desenvolvam, publiquem, implementem e gerenciem ambientes virtuais de ensino ${ }^{28}$. Apesar de terem surgido na década de 1990 em ambientes de ensino superior para implementar e manejar cursos a distância, as ferramentas de autoria têm sido cada vez mais utilizadas também para apoiar atividades presenciais e semipresenciais ${ }^{29}$.

As ferramentas de autoria podem oferecer espaços para a disponibilização de conteúdos e informações; interações dos alunos entre si e destes com seus professores; ambientes que facilitam o processo de ensino-aprendizagem, podendo acomodar diferentes estilos e objetivos de aprendizagem, estimulando a busca ativa e o compartilhamento de conhecimento; e recursos de gerência do processo educativo ${ }^{30}$. Oferecem, portanto, um ambiente de ensino, mediado pelo computador e constituído por uma combinação de ferramentas de software de natureza pedagógica, comunicacional e administrativa, integradas em um único sistema ${ }^{31}$.

Existem várias ferramentas de autoria disponíveis para a implementação de AVAs. O Moodle, por exemplo, que é uma ferramenta de uso livre e código aberto, é uma das mais conhecidas e usadas por diversas instituições públicas e privadas de ensino superior, apresentando sofisticação tecnológica e potencial para a criação e o gerenciamento de cursos na WEB $^{32}$. No Brasil, AulaNet ${ }^{33}$ e Teleduc $^{34}$ são ferramentas conhecidas por terem assumido uma abordagem de pesquisa em sua concepção ${ }^{35}$. Além da existência dessas ferramentas que integram diferentes recursos informacionais, comunicacionais e de gestão do ensino, com o avanço da WEB 2.0 o professor conta, atualmente, com uma série de facilidades para incorporar as TICs em suas práticas pedagógicas.

A introdução de TICs na educação médica pode ser caracterizada pelos seguintes objetivos: modificar as práticas pedagógicas, situando-as na perspectiva da educação permanente; preparar os futuros médicos para lidar com o novo perfil do paciente, que se informa muito mais facilmente em questões de saúde e doença pela Internet; e, por fim, capacitar os alunos para o uso de TICs, com foco em sua prática profissional, não apenas no âmbito do atendimento em saúde, mas também nas atividades de gestão ${ }^{20}$.

A pesquisa bibliográfica, especificamente sobre TICs e AVAs na educação médica, apontou muitos relatos de expe- riências de uso por diferentes disciplinas de áreas básicas e também de áreas clínicas para graduação, pós-graduação e educação continuada, tais como: ambientes virtuais de cirurgias e simuladores, de pacientes e casos clínicos e de modelos animais. Além disso, há relatos de uso das TICs como fonte de informação (apresentando textos, animações, imagens), como ferramenta de integração dos alunos (trabalhos em grupo, colaborativos, discussão de casos clínicos integrando conteúdos de áreas básicas) e como ferramenta de avaliação somativa e formativa (utilizando portfólios eletrônicos ou relatórios de atividades práticas) ${ }^{15,16,19,36-40}$. Há ainda experiências de ensino em cursos semipresenciais utilizando diversas abordagens pedagógicas simultaneamente ${ }^{41}$.

Cook, $\mathrm{J}^{42}$, em seu estudo sobre o uso de AVAs em diferentes cursos das ciências biomédicas, identificou que os ambientes são usados principalmente com o objetivo de fornecer informações sobre os cursos e disponibilizar conteúdo informativo. Resultados semelhantes encontraram Cook, DA et al. ${ }^{43}$ em uma revisão sistemática de estudos sobre intervenções baseadas na Internet, indicando uma variedade de configurações e métodos instrucionais nos AVAs, com um predomínio da disponibilização de textos e menor utilização de ferramentas comunicacionais.

Embora existam muitos trabalhos que descrevam os diferentes usos de TICs na educação médica, bem como os desafios desse processo de apropriação, a revisão da literatura revelou poucos estudos dedicados a caracterizar o perfil de utilização de ambientes virtuais de aprendizagem na formação médica em uma mesma instituição acadêmica.

\section{METODOLOGIA}

A presente pesquisa buscou identificar o perfil de utilização da ferramenta de autoria Constructore no contexto das atividades de ensino voltadas para diferentes níveis de formação médica na UFRJ. O universo dos cursos e disciplinas relacionados à formação médica na UFRJ, de um modo geral, configura-se da seguinte forma: o currículo médico de graduação contempla 42 disciplinas obrigatórias, das quais 24 (57\%) são da área de ciências clínicas, oito (19\%) de biociências, sete (17\%) interdisciplinares e três $(7 \%)$ da área de ciências humanas e sociais ${ }^{44}$. São nove os cursos de pós-graduação stricto sensu (mestrado e doutorado) ${ }^{45}$ e 81 os cursos lato sensu (residência médica e especialização) na área médica ${ }^{46}$

A Constructore (http://ltc.nutes.ufrj.br/constructore) é uma ferramenta de autoria de cursos/AVA desenvolvida pelo Núcleo de Tecnologia Educacional para a Saúde (NUTES) da UFRJ. Tal como em outras ferramentas de autoria, a estrutura da Constructore oferece vários recursos para criação, publica- 
ção e gerenciamento de cursos a distância e/ou semipresenciais. Um dos princípios básicos de concepção da Constructore é que a ferramenta seja facilmente compreendida pelo professor, possibilitando-lhe desenvolver seus materiais com autonomia, liberando-o para deter-se principalmente nos aspectos relacionados ao conteúdo, às estratégias de ensino e às suas formas de utilização nas práticas pedagógicas ${ }^{26}$.

Os primeiros ambientes de aprendizagem começaram a ser desenvolvidos na Constructore no primeiro semestre de 2007, por iniciativa pioneira de alguns docentes da área da saúde, que tomaram conhecimento da ferramenta por meio de convites e, posteriormente, por disseminação entre os próprios usuários. Até o final de 2010, a Constructore contava com 74 cursos da área das ciências da saúde, implementados por 35 professores, envolvendo um total de 4.105 alunos.

Para realizar este levantamento, foi delimitado o período entre o início de 2007 e o final do ano letivo de 2010, que corresponde aos quatro primeiros anos de implantação da ferramenta. Foram buscados dados sobre os AVAs implementados na Constructore por meio dos registros existentes na plataforma. O principal critério de seleção envolveu a identificação daqueles cursos oferecidos em diferentes contextos e níveis de formação médica (graduação, pós-graduação, educação continuada e extensão) nas unidades acadêmicas da UFRJ.

Com base nessa seleção, foram coletados os seguintes dados: número de cursos e suas edições (turmas); disciplinas e unidades acadêmicas envolvidas; número de professores e alunos envolvidos; utilização de diferentes recursos oferecidos pela ferramenta (conteúdos, comunicação e atividades). A análise dos dados foi organizada da seguinte forma: descrição geral dos cursos; configuração temática dos cursos; configuração da equipe docente; e utilização dos recursos pedagógicos.

A seguir, apresentamos os principais resultados obtidos, procurando traçar o perfil de utilização da ferramenta Constructore em processos de formação na área médica.

\section{ANÁLISE E RESULTADOS}

\section{Descrição geral dos cursos}

Nessa etapa, procurou-se traçar um perfil geral de utilização da ferramenta Constructore, identificando os cursos, os níveis de ensino, as unidades acadêmicas e as áreas do conhecimento em que os cursos se inserem. Além disso, observou-se o fluxo de uso da ferramenta ao longo do período estudado.

Como apresentado no Quadro 1, foram identificados 23 diferentes cursos que viabilizaram, no período de 2007-2010, um total de 63 AVAs na Constructore. Essa quantidade de AVAs explica-se pelo fato de um mesmo curso ter sido ofe- recido com mais de uma turma em um mesmo semestre ou por ter se repetido ao longo do período analisado. É evidente que esse é um número modesto quando levamos em conta a estrutura de uma instituição como a UFRJ e sua área da saúde. Porém, é preciso ressaltar que a integração de inovações em práticas educativas é um processo gradual e que exige mudanças que muitas vezes precisam de tempo e maturação para serem apropriadas, especialmente em contextos onde o foco do trabalho pedagógico do professor, por conta das pressões acadêmicas, torna-se secundário ${ }^{47,48}$.

Dentre os cursos existentes, identificou-se a seguinte distribuição de acordo com os níveis de ensino: 12 disciplinas (52\%) em nível de graduação, seis $(26 \%)$ de pós-graduação, quatro (17\%) cursos de formação continuada e um (5\%) de extensão. Um total de oito unidades acadêmicas situadas no Centro de Ciências da Saúde (n cursos $=20,87 \%$ ) e no Campus de Macaé (n cursos $=3,13 \%$ ) da UFRJ estão envolvidas na oferta de cursos semipresenciais. É interessante observar a distribuição de cursos oferecidos para o ensino médico pelas unidades acadêmicas do CCS/UFRJ na Constructore, em que apenas dois deles são diretamente oferecidos pela própria Faculdade de Medicina. Esse dado reflete, principalmente, a estrutura curricular dos cursos na área médica da UFRJ, que se organiza a partir da cooperação com várias unidades acadêmicas, e, ao mesmo tempo, coloca algumas questões sobre o possível desconhecimento do corpo docente sobre essa ferramenta e/ou a pouca disponibilidade para se dedicar a novas práticas educativas ${ }^{25}$. Cabe ainda ressaltar que o Campus de Macaé conta com três cursos (13\% do total) que utilizam a Constructore, o que pode ser interpretado de acordo com o fato de que sua formação médica conforma uma nova proposta educativa e estrutura curricular, bem como um corpo docente recém-integrado à UFRJ.

Do ponto de vista da evolução do uso da ferramenta Constructore (Quadro 2) ao longo do período estudado, observa-se que houve crescimento no número de cursos, docentes e alunos. Enquanto em 2007, primeiro ano de uso da Constructore, havia três professores, três cursos e um total de 133 alunos envolvidos; em 2010, estes números cresceram para 12 cursos, 25 professores e 681 alunos. Em 2010, houve a diminuição de um curso, acarretando também a diminuição do número de alunos em relação ao ano anterior. É importante analisar essa aparente estabilização em função de outros dados, como o contínuo aumento de professores envolvidos (em 2010 houve aumento de cinco docentes) e de novas edições dos mesmos cursos, indicando que, após um período inicial de crescimento e conhecimento da ferramenta, os docentes usuários podem ter promovido novas configurações pedagógicas e parcerias nos contextos dos cursos existentes. 
QuAdro 1

Títulos e códigos dos cursos analisados, suas unidades acadêmicas de origem e seus níveis de ensino

\begin{tabular}{|c|c|c|}
\hline Unidade Acadêmica da UFRJ & Código/Nome dos cursos & Nível de Ensino \\
\hline \multirow{7}{*}{$\begin{array}{l}\text { Núcleo de Tecnologia Educacional } \\
\text { para a Saúde (NUTES) } \\
\mathrm{N}=7\end{array}$} & C1 - Formação pedagógica de preceptores do internato médico & \multirow[t]{4}{*}{ Educação continuada } \\
\hline & C2 - Formação pedagógica na área da saúde & \\
\hline & C3 - Formação continuada de professores do polo de Macaé & \\
\hline & C4 - Preceptoria em atenção primária & \\
\hline & C5 - Metodologia científica & \multirow[t]{2}{*}{ Pós-Graduação } \\
\hline & C6 - Planejamento curricular e de ensino na área da saúde & \\
\hline & C7 - Aprendendo a aprender na área da saúde & Graduação \\
\hline \multirow{4}{*}{$\begin{array}{l}\text { Instituto de Bioquímica Médica (IbqM) } \\
\mathrm{N}=4\end{array}$} & C8 - Bioenergética para medicina & \multirow[t]{4}{*}{ Graduação } \\
\hline & $\begin{array}{l}\text { C9 - Integração Metabólica - atividades complementares - turmas } \\
\text { A/B/C/D }\end{array}$ & \\
\hline & C10 - Estrutura e função das proteínas & \\
\hline & C11 - Hormônios relacionados ao controle de peso corporal & \\
\hline \multirow{3}{*}{$\begin{array}{l}\text { Campus Macaé } \\
\mathrm{N}=3\end{array}$} & C12 - Biologia para saúde I & \multirow[t]{3}{*}{ Graduação } \\
\hline & $\begin{array}{l}\text { C13 - Capacitação de auxiliares de pesquisa para o estudo comorbidade } \\
\text { sofrimento psíquico álcool e drogas }\end{array}$ & \\
\hline & C14 - Clube científico de leitura "Saúde, doença e comunidade" & \\
\hline \multirow{3}{*}{$\begin{array}{l}\text { Instituto de Psiquiatria da UFRJ (IPUB) } \\
\mathrm{N}=3\end{array}$} & C15 - História e ética em saúde mental & \multirow[t]{2}{*}{ Pós-Graduação } \\
\hline & C16 - Metodologia em pesquisa qualitativa em saúde mental & \\
\hline & C17 - Reflexão sobre a prática médica & Graduação \\
\hline \multirow{2}{*}{$\begin{array}{l}\text { Instituto de Biofísica Carlos Chagas } \\
\text { Filho (IBCCF) } \\
\mathrm{N}=2\end{array}$} & C18 - Tópicos em neurofisiologia & Graduação \\
\hline & C19- Neuroanatomia & Pós-Graduação \\
\hline \multirow{2}{*}{$\begin{array}{l}\text { Hospital Universitário Clementino } \\
\text { Fraga Filho - Faculdade de Medicina } \\
\text { - Departamento de Cirurgia - } \\
\text { Anestesiologia } \\
\mathrm{N}=2\end{array}$} & C20 - Anestesiologia para internato & Graduação \\
\hline & C21 - Treinamento e qualificação profissional para o tratamento da dor & Extensão \\
\hline $\begin{array}{l}\text { Hospital Universitário Clementino } \\
\text { Fraga Filho - Faculdade de Medicina } \\
\text { - Departamento de Clínica Médica - } \\
\text { Nefrologia } \\
\mathrm{N}=1\end{array}$ & C22 - Glomerulonefrite & Graduação \\
\hline $\begin{array}{l}\text { Instituto de Puericultura e Pediatria } \\
\text { Martagão Gesteira } \\
\mathrm{N}=1\end{array}$ & C23 - Curso teórico para residentes do programa de pediatria & Pós-Graduação \\
\hline
\end{tabular}

\section{Configuração Temática dos Cursos — áreas do conhecimento}

Como já foi abordado anteriormente, a prática médica requer conhecimentos que envolvem diferentes áreas, como biociências e ciências clínicas, bem como ciências humanas e sociais. A análise da natureza das temáticas dos cursos oferecidos na Constructore apontou (Quadro 3) que eles se distribuem em todas essas áreas de conhecimento. Os sete cursos (30\%) na área de ciências humanas e sociais são aqueles oferecidos pelo Núcleo de Tecnologia Educacional para a Saúde (NUTES), para formação pedagógica e em pesquisa, em nível de pós-graduação e educação continuada. Os sete cursos (30\%) na área de biociências são oferecidos pelos Institutos de Biofísica $(n=2)$ e 
Bioquímica $(n=4)$, e pelo Campus de Macaé $(n=1)$, principalmente em nível de graduação $(n=6)$. São cinco os cursos $(22 \%)$ relacionados à área de ciências clínicas, sendo três em nível de graduação, um de pós-graduação e um de extensão. Os quatro cursos (17\%) categorizados na área interdisciplinar (dois de graduação e dois de pós-graduação) são oferecidos pelo Instituto de Psiquiatria $(n=3)$ e pelo Campus de Macaé $(n=1)$.

QUADRO 2

Evolução da utilização da Constructore no período de 2007-2010 (cursos, edições, docentes e alunos)

\begin{tabular}{|c|c|c|c|c|}
\hline Ano & $\begin{array}{c}\text { Número } \\
\text { de cursos }\end{array}$ & $\begin{array}{c}\text { Número } \\
\text { total de } \\
\text { edições }\end{array}$ & $\begin{array}{c}\text { Número de } \\
\text { professores }\end{array}$ & $\begin{array}{c}\text { Número } \\
\text { total de } \\
\text { alunos }\end{array}$ \\
\hline 2007 & 3 & 6 & 3 & 133 \\
\hline 2008 & 7 & 14 & 7 & 347 \\
\hline 2009 & 13 & 20 & 20 & 725 \\
\hline 2010 & 12 & 23 & 25 & 681 \\
\hline
\end{tabular}

\section{QUADRO 3}

Áreas de conhecimento, números/códigos dos cursos e unidades acadêmicas UFRJ

\begin{tabular}{|c|c|c|}
\hline $\begin{array}{c}\text { Áreas do } \\
\text { conhecimento }\end{array}$ & $\begin{array}{l}\text { N/código de } \\
\text { cursos }\end{array}$ & Unidades Acadêmicas UFRJ \\
\hline $\begin{array}{l}\text { Ciências } \\
\text { Humanas e } \\
\text { Sociais }\end{array}$ & $\begin{array}{l}7(\mathrm{C} 1, \mathrm{C} 2, \mathrm{C} 3 \\
\mathrm{C} 4, \mathrm{C} 5, \mathrm{C} 6 \text { e } \mathrm{C} 7)\end{array}$ & $\begin{array}{l}\text { Núcleo de Tecnologia } \\
\text { Educacional para a Saúde } \\
\text { (NUTES) }\end{array}$ \\
\hline \multirow[t]{3}{*}{ Biociências } & $\begin{array}{l}4(\mathrm{C} 8, \mathrm{C} 9, \mathrm{C} 10 \\
\text { e C11) }\end{array}$ & $\begin{array}{l}\text { Instituto de Bioquímica } \\
\text { Médica (IbqM) }\end{array}$ \\
\hline & 2 (C18 e C19) & $\begin{array}{l}\text { Instituto de Biofísica Carlos } \\
\text { Chagas Filho (IBCCF) }\end{array}$ \\
\hline & $1(\mathrm{C} 12)$ & Campus Macaé \\
\hline \multirow[t]{2}{*}{ Interdisciplinar } & $1(\mathrm{C} 14)$ & Campus Macaé \\
\hline & $\begin{array}{l}3(\mathrm{C} 15, \mathrm{C} 16 \mathrm{e} \\
\mathrm{C} 17)\end{array}$ & Instituto de Psiquiatria (IPUB) \\
\hline \multirow[t]{4}{*}{$\begin{array}{l}\text { Ciências } \\
\text { Clínicas }\end{array}$} & 2 (C20 e C21) & $\begin{array}{l}\text { Hospital Universitário } \\
\text { Clementino Fraga Filho } \\
\text { (HUCFF) - Faculdade de } \\
\text { Medicina - Departamento de } \\
\text { Cirurgia - Anestesiologia }\end{array}$ \\
\hline & $1(\mathrm{C} 13)$ & Campus Macaé \\
\hline & $1(\mathrm{C} 22)$ & $\begin{array}{l}\text { Hospital Universitário } \\
\text { Clementino Fraga Filho } \\
\text { (HUCFF) - Faculdade de } \\
\text { Medicina - Departamento de } \\
\text { Clínica Médica - Nefrologia }\end{array}$ \\
\hline & $1(\mathrm{C} 23)$ & $\begin{array}{l}\text { Instituto de Puericultura e } \\
\text { Pediatria Martagão Gesteira } \\
\text { (IPPMG) }\end{array}$ \\
\hline
\end{tabular}

Embora não se possa afirmar com base nos dados obtidos que haja alguma tendência mais marcante no uso das TICs em função dos conteúdos de aprendizagem das diversas áreas de conhecimento, essa distribuição relativamente equilibrada pode ser compreendida pela multiplicidade de possibilidades pedagógicas oferecidas pelos diferentes recursos e ferramentas das TICs, que atendem a diferentes abordagens, estilos e necessidades de ensino-aprendizagem ${ }^{27,28,47}$.

\section{Configuração do trabalho docente}

Os 23 cursos estudados e suas edições foram realizados por 44 diferentes professores e 24 tutores (14 alunos de pós-graduação, três alunos graduação que atuaram no curso e sete funcionários médicos). A composição das equipes de professores apresentou ampla variabilidade, com envolvimento de docentes $(n=25)$, alunos de pós-graduação $(n=9)$ e funcionários técnico-administrativos da saúde da UFRJ (seis médicos, três psicólogos e um fisioterapeuta), atuando isoladamente ou em cooperação com outros docentes, funcionários ou alunos de pós-graduação. A maioria dos professores responsáveis ( $\mathrm{n}=$ $25,57 \%$ ) eram docentes, enquanto a maioria dos tutores eram alunos de pós-graduação ( $\mathrm{n}=14,58 \%$ ); entretanto, observou-se a participação de funcionários da área da saúde como responsáveis pelos cursos $(n=6,14 \%)$ ou como tutores $(n=7$, $50 \%$ ). Muitos deles participaram de mais de uma edição de um determinado curso $(\mathrm{n}=18,41 \%)$, ou mesmo de diferentes cursos ( $n=12,27 \%$ ), em equipes com diferentes configurações; outros tiveram apenas uma única participação ( $n=20,45 \%$ ).

Independentemente das motivações para essa variabilidade na conformação das equipes docentes, deve-se salientar a flexibilidade da ferramenta de autoria, que permite que os professores se organizem de diferentes formas, de acordo com suas necessidades, viabilizando uma docência colaborativa. Várias pesquisas têm mostrado que a colaboração docente, além de constituir-se como apoio mútuo para enfrentar as dificuldades cotidianas dos professores e para o aprimoramento de suas práticas, pode contribuir para o diálogo interdisciplinar, para a consolidação da colegialidade e para o desenvolvimento de currículos mais integradores ${ }^{49,50,51}$.

\section{Utilização dos recursos pedagógicos}

A descrição dos recursos pedagógicos utilizados nos cursos que adotaram a Constructore encontra-se nos gráficos 1, 2 e 3 . É importante lembrar que todos os 23 cursos analisados eram presenciais, fazendo uso da ferramenta como complemento às suas atividades em sala de aula, em laboratórios ou mesmo em serviços de saúde. Nenhum desses cursos se propunha a ser totalmente a distância, configurando uma tendência ao mode- 
lo semipresencial do ensino superior, garantido pela legislação brasileira, que autoriza a carga horária de até $20 \%$ do total do curso com atividades a distância ${ }^{52}$. Essa tendência é também revelada em outros estudos em contextos internacionais ${ }^{28,29,53}$.

Em relação aos recursos de conteúdo utilizados (Gráfico 1), observou-se que houve a disponibilização de materiais de leitura para download sob a forma de textos ( $n=20,87 \%)$ e de apresentações de slides ( $n=10,43 \%$ ), assim como oferta de links ( $n=12,52 \%)$ e bibliografia $(n=7,30 \%)$ para consulta, formatos esses habitualmente utilizados nas práticas de ensino presencial. O uso de animações, vídeos e/ou imagens ocorreu em três cursos (13\%). Portanto, os AVAs parecem ter sido mais utilizados para disponibilizar conteúdo textual e menos para explorar o potencial das TICs para a representação de informações visuais, sejam elas em imagens estáticas ou dinâmicas em diferentes mídias, tal como relatado por outros autores $^{42,43}$. Vários estudos relatam que esses recursos multimídia contribuem de forma efetiva para a aprendizagem de conceitos abstratos e de difícil visualização, bem como de processos que se desenvolvem ao longo do tempo, impossíveis de serem observados, acompanhados e controlados em situações reais ${ }^{19}$, 36,54 . Tais conceitos e processos são inerentes aos conhecimentos das ciências básicas e clínicas e, portanto, importantes para a educação médica ${ }^{18,55}$.

\section{GRÁfICO 1}

Recursos de conteúdo disponibilizados pelos cursos

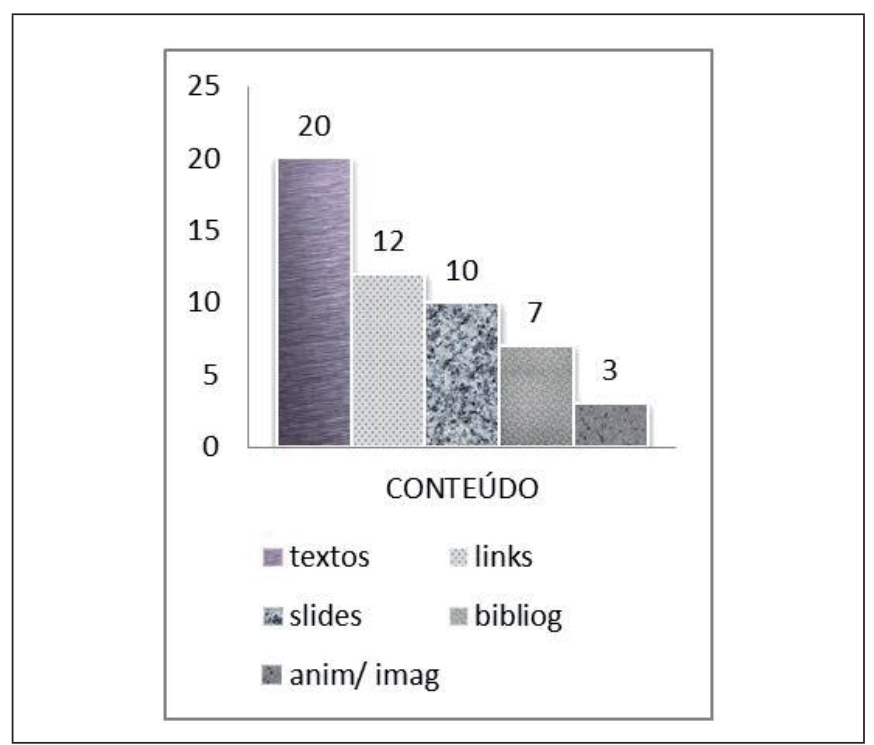

Sabe-se que uma das potencialidades do uso da informática e da Internet na educação é a possibilidade de armazenar e tornar disponível grande quantidade de informação, prova- velmente substituindo a tradicional pasta de leituras para os alunos fazerem cópias, e isso pode constituir uma forma ágil e econômica de tornar os materiais acessíveis aos alunos ${ }^{5,56}$. Sabe-se também que a Internet constitui uma rede abrangente, um enorme repositório de recursos que colocam desafios para a incorporação de uma postura crítica e analítica dos sujeitos diante do emaranhado de informações disponíveis e, portanto, impõe novas demandas para a formação de profissionais da saúde, capazes de lidar com discernimento perante esse dinâmico e saturado contexto informacional em suas práticas e tomadas de decisões ${ }^{57}$. Nesse sentido, nos cursos analisados, essas questões aparentemente não foram consideradas pelos professores aos criarem seus AVAs.

\section{GrÁfico 2}

Recursos comunicacionais utilizados pelos cursos

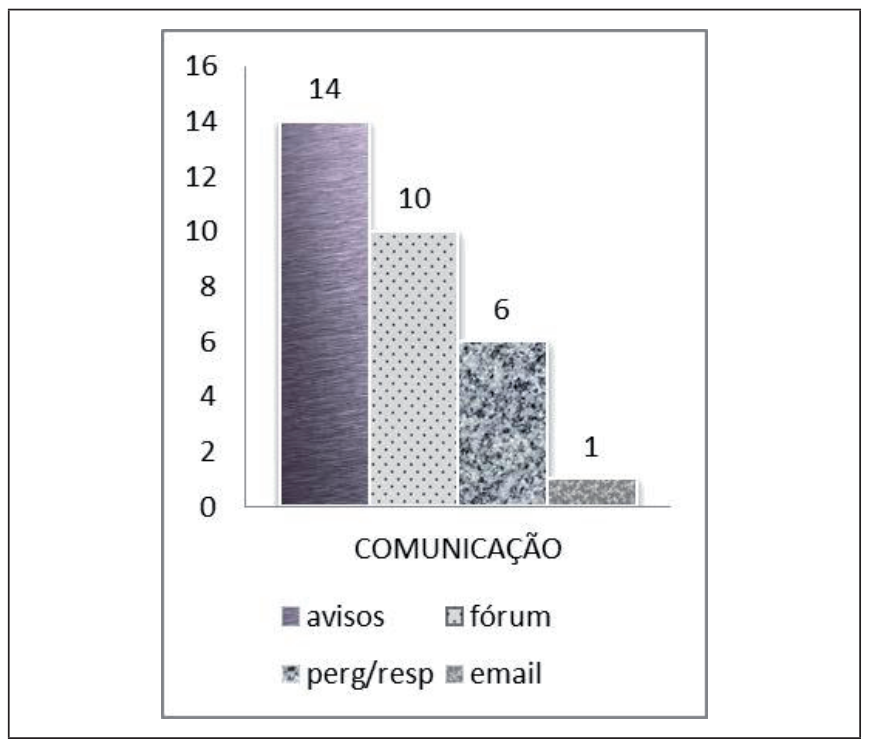

Em relação aos recursos de comunicação utilizados (Gráfico 2), 17 cursos (74\%) adotaram pelo menos uma das ferramentas de comunicação assíncrona disponíveis na Constructore (e-mail, fórum, avisos, perguntas e respostas). O recurso mais utilizado foi o do quadro de avisos ( $\mathrm{n}=14,61 \%$ ), em que professores fazem alertas e comunicam aspectos referentes à rotina e à administração do curso aos alunos. $\mathrm{O}$ uso do fórum como espaço para expressão de opiniões, elaborações por parte dos alunos acerca do conteúdo em discussão e comentários dos professores sobre essa produção foi adotado em seis cursos (26\%) (C1, C2, C3, C5, C6 e C21), a maioria deles da área de ciências humanas. Em quatro cursos (17\%) (C9, C10, C11 e C20), os fóruns foram usados como espaço de perguntas e respostas, para esclarecer questões mais imedia- 
tas e práticas relacionadas ao curso. A ferramenta de e-mail oferecida pela Constructore para uso interno aos AVAs não foi praticamente utilizada. Assim como os dados apresentados nesta pesquisa, alguns estudos já apontaram uma tendência do uso de recursos de comunicação em cursos on-line serem principalmente para trocas de informações pontuais sobre questões consideradas mais administrativas (perguntas sobre datas, entregas de trabalho, pedidos de orientações pontuais, etc.) em vez de explorar o potencial de comunicação para estabelecer uma relação dialógica e colaborativa no processo de aprendizagem ${ }^{58}$. Há outras questões relatadas na literatura a serem ponderadas no que diz respeito à moderada utilização dos recursos de comunicação on-line: de um modo geral, intervenções por escrito implicam em preocupações com a própria escrita e com a redação das respostas enviadas, ou seja, terminam por acarretar em sobrecarga de trabalho para os professores ${ }^{25}$.

\section{GRÁFICO 3}

Tipos de atividades propostas pelos cursos

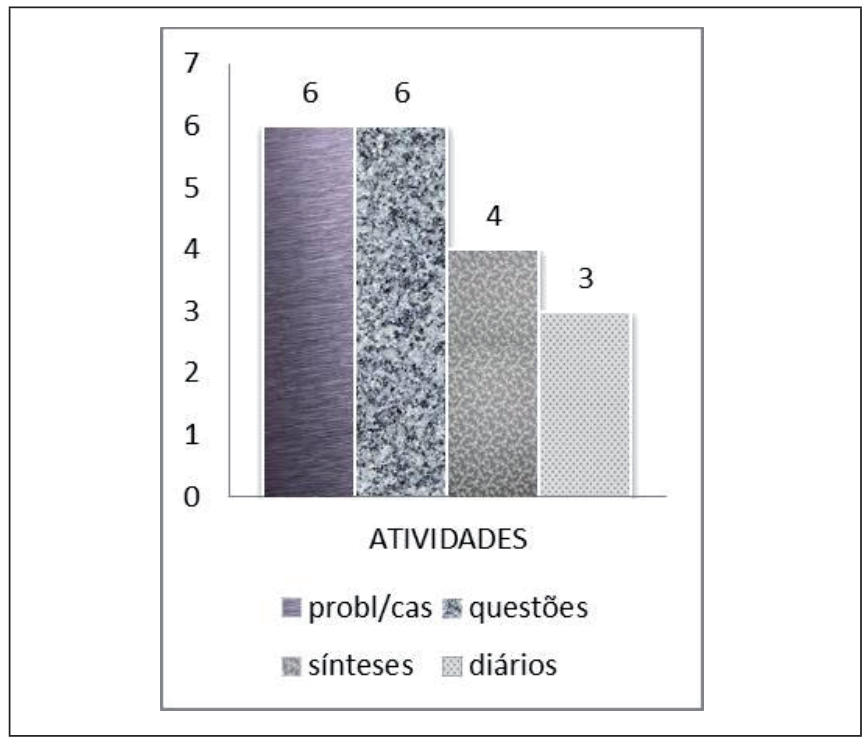

No que diz respeito às atividades de aprendizagem on-line (Gráfico 3), 17 cursos (74\%) apresentaram propostas de atividades para os alunos desenvolverem em seus AVAs. Em seis cursos (26\%) (C2, C9, C10, C12, C21, C23), a principal atividade constava da resolução de problemas ou casos, envolvendo os estudantes na busca ativa por materiais na Internet, nos quais pudessem basear suas reflexões e propostas de soluções. Outros seis cursos (26\%) (C4, C6, C11, C13, C15, C22) desenvolveram questionários a serem respondidos após leitura de material textual disponibilizado nos AVAs. A soli- citação de produção teórica individual ou em trabalhos em grupo (sínteses individuais e coletivas) foi proposta em cinco cursos $(17 \%)(\mathrm{C} 1, \mathrm{C} 3, \mathrm{C} 6, \mathrm{C} 12)$, todos de uma mesma unidade acadêmica (NUTES), na área de ciências humanas e, em sua maioria, voltados para a formação pedagógica de profissionais de saúde. Três cursos (13\%) (C5, C7, C20) propuseram aos alunos a produção de relatórios reflexivos baseados em atividades práticas. Verificou-se uma diversidade de atividades que demandam reflexão, pesquisa e elaboração de textos pelos alunos. O professor tem, assim, a possibilidade de lançar mão do ambiente informatizado para facilitar o acesso e a correção dos trabalhos dos alunos, propondo atividades que combinam, em diferentes formatos, reflexão e pesquisa na Web.

Um dado interessante foi que os cursos realizados em mais de uma edição $(n=9)$ apresentaram formas de utilização dos recursos que variaram de acordo com o professor responsável, mesmo em atividades que aconteceram simultaneamente, e para um mesmo professor, em diferentes semestres em que este ofereceu o curso. É possível sugerir que fatores ligados aos docentes, não apenas aqueles ligados a suas características individuais, mas também aqueles relacionados às pressões do cotidiano, mais variáveis ao longo de um curto tempo, possam interferir na forma de utilização das TICs. O fato é que a ferramenta de autoria utilizada permite essa flexibilidade, mesmo em replicações de um mesmo curso.

A avaliação do curso ou a autoavaliação de desempenho pelos alunos não foi disponibilizada em nenhum dos AVAs analisados, apesar de haver na ferramenta Contructore facilidades para construção de formulários para esse fim. A avaliação é uma das "instituições" mais consolidadas no ensino formal, sendo elemento conformador da cultura que permeia as práticas educativas na universidade ${ }^{59}$. É possível que os professores ainda não tenham experimentado maior conhecimento e confiança em um sistema informatizado de avaliação, mesmo que formativo. A própria legislação brasileira sobre Educação a Distância ${ }^{52}$ indica que as avaliações nessa modalidade de ensino, para serem válidas, devem ser presenciais.

Para Sugrue ${ }^{56}$, os usos da Internet no processo de ensino-aprendizagem podem ser categorizados como: acesso e organização da informação, relacionado ao uso das TICs como repositório de informações; atividades autênticas, envolvendo a participação ativa dos alunos na articulação dos conhecimentos teóricos e práticos; aprendizagem colaborativa, referindo-se à construção social do conhecimento, ou seja, à mediação dos processos que envolvem a aprendizagem em coletividade; e acompanhamento e avaliação dos alunos, abrangendo 
as estratégias para acompanhar e avaliar o desenvolvimento cognitivo dos alunos ${ }^{26}$. Assim, a partir dos dados obtidos neste levantamento sistemático, pode-se afirmar que a tendência dos usos da Internet concentrou-se, principlamente, em duas dessas categorias: acesso e organização da informação e atividades autênticas, que contemplaram mais da metade das atividades propostas.

\section{CONCLUSÕES}

Ainda que a experiência com a ferramenta de autoria de AVAs Constructore seja relativamente moderada no contexto do grande número de disciplinas de graduação, pós-graduação e de cursos de educação continuada relacionados ao ensino médico e esteja inserida no contexto de uma universidade pública como a UFRJ, torna-se pertinente a compreensão sobre as principais características do perfil de uso dessas experiências pioneiras de atividades semipresenciais, tendo em vista a formulação de programas futuros que procurem oferecer aos docentes infraestrutura e suporte tecnológico, bem como apoio pedagógico no sentido de promover o uso de TICs nas práticas educativas. Até o momento da condução desta pesquisa, não havia no âmbito institucional da UFRJ, nem na área da saúde propriamente dita, qualquer programa oficial com esse objetivo.

Em geral, a iniciativa de incorporar o uso de TICs por meio da ferramenta Contructore pelos professores do ensino médico ocorreu prioritariamente nas disciplinas de graduação e nas de pós-graduação, o que caracteriza que, em geral, o uso foi direcionado principalmente para o ensino regular, incorporando, de fato, o desafio de novas práticas ao contexto dos cursos tradicionais da área. Foi constatado também que foram principalmente as disciplinas oferecidas pelas unidades parceiras da Faculdade de Medicina aquelas que mais utilizaram os recursos de TICs. Isso indica que os docentes das áreas básicas e das ciências humanas e sociais, muito mais do que os da área clínica, têm participado dessa iniciativa.

A flexibilidade da ferramenta de autoria permitiu ampla variabilidade na configuração das equipes docentes ao longo do tempo em um mesmo curso ou por um mesmo professor e seus parceiros. Essa configuração potencializou os arranjos existentes e já praticados na institução, para superar as dificuldades impostas pela sobrecarga de sala de aula e o grande número de alunos que, em geral, são divididos em grupos para o melhor andamento do trabalho pedagógico. Mesmo não sendo a motivação central, essas necessidades terminam aportando benefícios às práticas educativas colaborativas e interdisciplinares ${ }^{51}$.

No que diz respeito à utilização de recursos de conteúdo em seus AVAS, os professores privilegiaram a disponibilização de informação textual em relação a outros recursos midiáticos, tais como: imagens, animações e vídeos, com potencial para representação de fenômenos e processos biológicos presentes no currículo médico. Entre os recursos de comunicação disponíveis, o quadro de avisos foi o mais utilizado. Mesmo quando o fórum foi implementado, sua finalidade foi servir de espaço para tirar dúvidas sobre vários aspectos relacionados aos requisitos e funcionamento dos cursos/disciplinas. Portanto, o potencial de comunicação e troca entre professores e alunos e entre os alunos foi praticamente inexplorado. As atividades de ensino-aprendizagem propostas nos AVAs centraram-se na reflexão e na ação do aluno, algumas envolvendo estratégias como ensino baseado em casos com pesquisa na Web, e outras requisitando o aluno a elaborar suas próprias observações reflexivas a partir de textos disponibilizados. As facilidades oferecidas pela Constructore para elaborar diferentes avaliações on-line não foram usadas em nenhum dos AVAs construídos pelos professores.

Sabe-se que a incorporação de inovações no ambiente de ensino se inicia geralmente com pouca alteração de sua dinâmica habitual ${ }^{47}$. À semelhança do que vemos relatado em pesquisas realizadas no Brasil e em outros países, observamos neste estudo uma tendência dos professores, ao adotarem as TICs, de reproduzirem suas práticas presenciais ${ }^{43,48}$. Para Ertmer ${ }^{60}$, a integração de TICs na educação deve ser consistente com os valores, crenças e práticas pedagógicas correntes.

Apesar da grande expectativa em torno do uso de TICs na promoção de mudanças no ensino médico, buscando atender os desafios atuais da formação nessa profissão, é importante compreender algumas questões relacionadas à integração de TICs no contexto educacional e que podem exercer influência sobre o perfil delineado a partir do estudo a partir das disciplinas e cursos relacionados ao ensino médico que adotaram a ferramenta de autoria Constructore.

Se por um lado o processo de ensino-aprendizagem compreende um fenômeno extremamente complexo e dependente do contexto e dos sujeitos envolvidos em sua prática ${ }^{61}$. Por outro, as tecnologias devem ser compreendidas como ferramentas criadas pelo conhecimento humano para resolver problemas e desenvolver produtos; portanto, suas potencialidades e limitações são definidas de acordo com tais necessidades. As TICs não foram concebidas como recursos educacionais. Assim, seu uso no processo de ensino-aprendizagem requer o redirecionamento de sua funcionalidade original, de forma que possam ser adaptadas para fins e necessidades pedagógicos ${ }^{62}$.

Isso coloca desafios para os professores em geral e, mais especificamente, para os da área das ciências da saúde, que, além da sobrecarga de trabalho e de raras oportunidades para refletir sobre sua prática pedagógica, do papel secundário 
dado às atividades docentes quando comparadas às atividades de pesquisa na universidade ${ }^{63,64}$, da falta de formação para a docência ${ }^{49,64}$, precisam transitar entre esses dois "mundos" — o da pedagogia e o da tecnologia — onde as normas, valores e a linguagem são tão diversos.

As TICs possuem grande potencial para desenvolver modelos e processos educativos integradores. No entanto, por si só não são capazes de resolver as demandas e os problemas da formação em saúde: isso depende da abordagem pedagógica e da visão de educação, de tecnologia e de saúde daqueles que as adotam. Dessa forma, é fundamental oferecer aos professores da área da saúde experiências pautadas na disponibilização de tecnologias fáceis de serem compreendidas e utilizadas, contando com apoio tecnológico e pedagógico para que eles possam se concentrar nos desafios da formação médica com flexibilidade e criatividade para superar as barreiras desse complexo processo. Além disso, pesquisas futuras deverão aprofundar as questões levantadas a partir dos resultados deste estudo, com base na perspectiva dos próprios docentes e alunos envolvidos nas experiências.

\section{REFERÊNCIAS}

1. Hall $P$, Weaver L. Interdisciplinary education and teamwork: a long and winding road. Med Educ. 2001;35(9):867-75.

2. Struchiner M, Gianella TR, Ricciardi RMV. Novas tecnologias de informação e educação em saúde diante da revolução comunicacional e informacional. In: Minayo MCS, Coimbra Junior CEA, (Org.). Críticas e atuantes: ciências sociais e humanas em saúde na América Latina. Rio de Janeiro: Fiocruz; 2005. p. 257-72.

3. Araújo D, Miranda M, Brasil S. Formação de profissionais de saúde na perspectiva da integralidade. Rev Bahiana Saúde Pública. 2007;31(supl 1):20-31.

4. Santana J, Campos F, Sena R. Formação profissional em saúde: desafios para a universidade. CADRHU [on-line].2004;233-44. [acesso em 8 jun 2012]. Disponível em: http:/ /www.opas.org.br/rh/publicacoes/textos_apoio/ ACF2114.pdf

5. Cooke M, Irby DM, Sullivan W, Ludmerer K. American Medical Education 100 Years After The Flexner Report. N Engl J Med. 2006;355(13):1339-44.

6. Oliveira NA. Ensino médico no Brasil: desafios e prioridades, no contexto do SUS - um estudo a partir de seis estados brasileiros. Rio de Janeiro; 2007. Doutorado [Tese] - Fundação Oswealdo Cruz .

7. Rego S. Educação médica: história e questões. In: Rego S. Formação ética dos médicos: saindo da adolescência com a vida (dos outros) nas mãos. Rio de Janeiro: Fiocruz; 2003.
8. Pagliosa FL, da Ros M A. O relatório Flexner: para o bem e para o mal. Rev Bras Educ Med. 2008;32(4):492-9.

9. Spencer, J. A; Jordan, R. K. Learner centred approaches in medical education. BMJ (Clinical research ed.), 1999;318(7193):1280-3.

10. Feuerwerker LCM. O movimento mundial de educação médica: as conferências de Edinburgh. Cad ABEM. 2006;2:30-38.

11. Aguilar-Da-Silva R, Perim G, Abdala I. Abordagens pedagógicas e tendências de mudanças nas escolas médicas. Rev Bras Educ Med. 2009;33(supl 1):53-62.

12. Batista SH. Aprendizagem, ensino e formação em saúde: das experiências às teorias em construção. In: Batista $\mathrm{N}$, Batista SH, (Org). Docência em saúde: temas e experiências. São Paulo: Senac; 2004. p. 57-74.

13. Ceccim RD, Carvalho YM. Ensino da saúde como projeto da integralidade: a educação dos profissionais de saúde no SUS. In: Pinheiro RRB, Ceccim RAM. Ensinar saúde: a integralidade e o SUS nos cursos de graduação na área da saúde. Rio de Janeiro: IMS/UERJ-CEPESC-ABRASCO; 2006. p. 69-92.

14. Ceccim RD, Feuerwerker L. Mudança na graduação das profissões de saúde sob o eixo da integralidade. Cad Saúde Pública. 2004;20(5):1400-10.

15. Ward JPT, Gordon J, Field MJ, Lehman HP. Communication and information technology in medical education. Lancet. 2001;357:792-6.

16. Stern S. Computer-assisted medical education: current and potential roles. Perspectives Biol Med. 2008;51(1):22-30.

17. Bliuc AM, Goodyear P, Ellis RA. Research focus and methodological choices in studies into students' experiences of blended learning in higher education. Internet Higher Educ. 2007;10(4):231-44.

18. Espíndola MB, El-Bacha T, Gianella TR, Struchiner M, Silva WS, Poian AT. Teaching energy metabolism using scientific articles: Implementation of a virtual learning environment for medical students. Biochem Mol Biol Educ. 2010;38(2):97-103.

19. Hogarth ME, Sabbatini RME. Informática e a medicina do século XXI. Informática Médica [on-line], 1998;1(2) [acesso em 30 jan. 2010]. Disponível em: <http:/ / www.informaticamedica.org.br/informaticamedica/n0102/hogarth.htm>

20. Karsenti T, Charlin B. Information and Communication Technologies (ICT) in Medical Education and Practice: The Major Challenges. Revue Internationale des Technologies en Pédagogie Universitaire, 2008;2:68-81.

21. Trindade MAB, (Org). As tecnologias da informação e comunicação (TIC) no desenvolvimento de profissionais do 
Sistema Único de saúde (SUS). São Paulo: Instituto da Saúde; 2011. 266p.

22. Wen CL. Telemedicina e Telessaúde: aplicação de tecnologia para promover educação interativa e formação de rede de interconsulta profissional em saúde. In:Trindade MAB, (org.). As tecnologias da informação e comunicação (TIC) no desenvolvimento de profissionais do Sistema Único de saúde (SUS). São Paulo: Instituto da saúde; 2011. p. 95-112.

23. Dahmer A, Flores C, Pinheiro L, Pinto M. A literatura e a diversidade midiática como estratégias pedagógicas na educação a distância em saúde: o projeto UNA-SUS/ UFCSPA. ESUD - VIII Congresso Brasileiro de Ensino Superior a Distância [Internet]. 2011 [cited 2013 Feb 28]. p. 1-19. Available from: http://lite.dex.ufla.br/esud2011/ images/abook_file/91848.pdf

24. Mckimm J, Jollie C, Cantillon P. Web-based learning. British Med J. 2003;326:870-3.

25. Struchiner M, Gianella TR, Espindola MB. Technological Pedagogical Content Knowledge of University Professors who Adopted an Online Authoring Tool in Their Educational Practices: A Case Study of Blended Learning Experiences in Health Science Education. In: Verbraeck A, Helfert M, Cordeiro J, Shishkov B, (Eds.): CSEDU 2011 - Proceedings of the 3rd International Conference on Computer Supported Education, Noordwijkerhout; 2011. p.56-64.

26. Struchiner M. Apreciação analítica de ambientes construtivistas de aprendizagem baseados em novas tecnologias de informação e de comunicação para a educação na área das ciências da saúde. Projeto submetido ao CNPq para renovação de Bolsa em Produtividade em Pesquisa (PQ), 2009.

27. Giannella TR, Struchiner M. Construção e aplicação de um modelo de análise de materiais educativos baseados na Internet para o ensino de ciências e saúde. REEC Revista Eletrónica de Enseñanza de las Ciencias. 2010;9:530-48.

28. Morgan G. Faculty use of course management systems. Colorado: ECAR, EDUCAUSE Center for Applied Research; 2003. 97p.

29. Rajapakse S, Fernando D, Rubasinghe N, Gurusinghe S. E-Learning in medical education: Guide supplement 32.6--Practical application. Med Teac. 2009;31(5):452-3.

30. Britain SA. Framework for Pedagogical Evaluation of Virtual Learning Environments. Report 41. Bangor, RU: Joint Information Systems Committee of the Higher Education Funding Councils; 1999.

31. Ellaway, R.H. Evaluating a Virtual Learning Environment in Medical Education. Edinburgh; 2005. Doutorado [Tese] - University of Edinburgh.
32. Reilly CA, Willians JJ. The price of free software: labor, ethics and context in distance education. Computers and Composition. 2006;23(1):68-90.

33. Lucena CJP, Fuks H. Educação na era da Internet. Rio de Janeiro: Clube do Futuro; 2000.

34. Tessarollo MR. Ambiente de autoria de cursos a distância (AutorWeb). Campinas; 2000. Mestrado [Dissertação] - : Universidade Estadual de Campinas.

35. Giannella TR. Inovações no ensino das ciências e da saúde: pesquisa e desenvolvimento da ferramenta Constructore e do Banco Virtual de Neurociência. Rio de janeiro; 2007. Doutorado [Tese] - Universidade Federal do Rio de Janeiro.

36. Sabbatini RME. Internet em Medicina: os recursos. Informática Médica [on-line], 1998;1(1).[acesso em 30 jan. 2010] Disponível em: <http://www.informaticamedica.org.br/ informaticamedica/n0101/sabbatini.htm>

37. Kitt S. Becoming a physician: teaching anatomy in the digital world. New Engl J Med. 2004;13:1279-81.

38. Valcked M,Wever B. Information and communication technologies in higher education: evidenced-based practice in medical education. Med Teach. 2006;28(1):40-8.

39. Cook DA. Where are we with Web-based learning in medical education? Med Teach. 2006;28(7):594-8.

40. Debolt D. Can virtual patients help real professors teach medicine? The chronicle of Higher Education, 2008;55:16.

41. Shaffer K, Small JE. Blended learning in medical education: use of an integrated approach with web-based small group modules and didactic instruction for teaching radiologic anatomy. Academic Radiology, 2004;11(9):1059-1070.

42. Cook J, Institute for Learning and Research Technology University of Bristol. Review of virtual learning environments in UK Medical, Dental and Veterinary Education. RU: The Higher Education Academy. 2003, 85p. ( Special Report 6.)

43. Cook DA, Garside S, Levinson AJ, Dupras DM, Montori VM. What do we mean by web-based learning? A systematic review of the variability of interventions. Med Educ. 2010;44(8):765-74.

44. Universidade Federal do Rio de Janeiro.Faculdade de Medicina. Graduação. Curso de Medicina. [homepage na Internet]. Rio de Janeiro: UFRJ. [acesso em 8 jun. 2012]. Disponível em: http://www.medicina.ufrj.br/colchoes. php?id_colchao $=43$

45. Universidade do Rio de Janeiro. Pró-Reitoria de Pós-Graduação e Pesquisa. Cursos. Stricto Sensu. Ciências da Saúde. [homepage na Internet]. Rio de Janeiro:UFRJ. [acesso em 8 jun. 2012] Disponível em: http:/ / www.pr2.ufrj.br/ 
46. Pró-reitora de Pós-Graduação (PR2). Cursos, Lato Sensu. Ciências da Saúde. [homepage na Internet]. Rio de Janeiro: Universidade Federal do Rio de Janeiro. [capturado em 8 jun. 2012]. Disponível em: http:/ / www.pr2.ufrj.br/

47. Nachmias R. A research framework for the study of a campus-wide Web-based academic instruction Project. Internet Higher Educ. 2002;5(3):213-29. DOI: 10.1016/S10967516(02)00103-3

48. Leite MTM, Carlini AL, Ramos MP, Sigulem D. Educação médica continuada on-line: potencial e desafios no cenário brasileiro. Rev Bras Educ Med. 2010;34(1):141-9.

49. Santomé JT. Globalização e interdisciplinaridade: o currículo integrado. Porto Alegre: Artmed. 1998.

50. Fullan M, Hargreaves A. A escola como organização aprendente: buscado uma educação de qualidade. 2 ed. Porto Alegre: Artmed; 2000. 136p.

51. Tractenberg LE, Struchiner M. A emergência da colaboração na educação e as transformações na sociedade pósindustrial: em busca de uma compreensão problematizadora. Boletim Técnico do SENAC. 2010;32(2):65-77.

52. Brasil. Ministério da educação. Portaria $n^{\circ} 4.059$, de 10 de dezembro de 2004. regulamenta modalidade semi-presencial. Diário Oficial União, Brasília, 13 dez 2004, Seção 1, p. 34.

53. Harrington CF, Gordon SA, Schibik TJ. Course management system utilization and implications for practice: A national survey of department chairpersons. Online J Distance Learn Adm. 2004;7(4):1-14.

54. Macaulay JO, Van Damme MP, Walker KZ. The use of contextual learning to teach biochemistry to dietetic students, Bio-chem Mol Biol Educ. 2009;37:137-42.

55. Groves H. Innovations in rehabilitation sciences education: preparing leaders for the future. New York: Springer;2005. Cap. Strategies for integrating Basic Sciences in Curriculum. p. 23-27.

56. Sugrue B. Cognitive Approaches to Web-Based Instruction. In: Lajoie S, (Ed.) Computers as Cognitive Tools: No More Walls. Mahwah, New Jersey: Lawrence Erlbaum Associates; 2000. p133-62.

57. Struchiner M, Giannella TR. Saber docente: o domínio do conhecimento e a competência pedagógica. In: Struchiner M. Giannella TR. Aprendizagem e prática docente na área da saúde: conceitos, paradigmas e inovações. Washington DC: OPS; 2005. p.9-16.
58. Heidrich DB, Angotti JA. P. Implantação e avaliação de ensino semipresencial em disciplinas de bioquímica utilizando ambiente virtual de aprendizagem. Rev Bras Ensino Bioquímica Bio Molecular. 2010;1:p E1-E14.

59. Cunha MI. Docência na universidade, cultura e avaliação institucional: saberes silenciados em questão. Rev Bras de Educ. 2006;11(32):258-71.

60. Ertmer PA. Teacher pedagogical beliefs: The final frontier in our quest for technology integration? Educ Technol Res Development. 2005;53(4):25-39.

61. Spiro RJ, Jehng JC. Cognitive flexibility and hypertext: theory and technology for the nonlinear and multidimensional traversal of complex subject matter. In: Nix D, Spiro R, (Eds.). Cognition, education and multimedia: Exploring ideas in high technology. Hillsdale, NJ: Lawrence Erlbaum; 1990. p.163-205.

62. Mishra P, Koehler MJ. Technological pedagogical content knowledge: a framework for teacher knowledge. Teachers College Report.2006;108:1017-54.

63. Costa N. Docência no ensino médico: por que é tão difícil mudar? Rev Bras Educ Med. 2005;29(1):21-30.

64. Medeiros AMS. Docência no ensino superior: dilemas contemporâneos. R. Faced. 2007;12:71-87.

\section{CONTRIBUIÇÃO DOS AUTORES}

Ekaterini S. Goudouris, participou da coleta e análise dos dados, elaboração do artigo, Taís R. Giannella participou na análise dos dados e na redação do artigo, Miriam Struchiner participou no desenvolvimento da pesquisa sobre a integração de TICs nas práticas educativas na universidade, orientação da pesquisa e participação na análise e na redação do artigo.

\section{CONFLITO DE INTERESSES}

Declarou não haver.

\section{ENDEREÇO PARA CORRESPONDÊNCIA}

Ekaterini S. Goudouris

Rua professor Luis Cantanhede, 77 — apto. 101

Laranjeiras - Rio de Janeiro

CEP. 22245-040 RJ

E-mail: egoudouris@gmail.com 